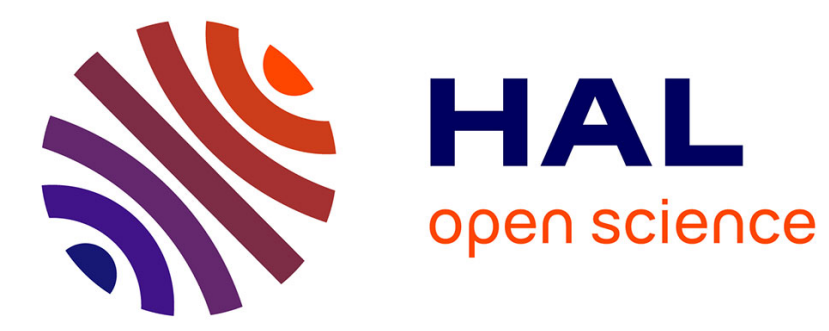

\title{
Figures de la science dans des contextes de conception
} Annie Gentes, Justine Peneau

\section{To cite this version:}

Annie Gentes, Justine Peneau. Figures de la science dans des contextes de conception. Communication \& langages, 2020, Cultures numériques en afriques francophone. Observer les pratiques, questionner les concepts, 205, pp.147-165. 10.3917/comla1.205.0147 . hal-03120684

\section{HAL Id: hal-03120684 https://hal.science/hal-03120684}

Submitted on 13 Apr 2021

HAL is a multi-disciplinary open access archive for the deposit and dissemination of scientific research documents, whether they are published or not. The documents may come from teaching and research institutions in France or abroad, or from public or private research centers.
L'archive ouverte pluridisciplinaire HAL, est destinée au dépôt et à la diffusion de documents scientifiques de niveau recherche, publiés ou non, émanant des établissements d'enseignement et de recherche français ou étrangers, des laboratoires publics ou privés. 


\title{
FIGURES DE LA SCIENCE DANS DES CONTEXTES DE CONCEPTION
}

\author{
Annie Gentès et Justine Peneau
}

\section{RÉSUMÉ}

Nous nous intéressons aux enjeux de la représentation de la science dans des milieux de conception à partir d'une filature de trois ans de deux physiciens impliqués dans des dispositifs de conception. Notre hypothèse est que les scientifiques y modulent leur posture et y affirment certains aspects de la science en adoptant plusieurs « rôles ", au sens de Goffman, afin de permettre de nouvelles collaborations entre les disciplines. Cette analyse permet de repérer des formes de dramaturgie de la science, au sens de Habermas, au travers de cinq « figures » tirées des discours des deux physiciens : « le professeur universitaire », " l'honnête homme », « l'expert », « le chercheur et le scientifique-créateur ». Nous observons que ces figures sont représentatives mais plus encore, qu'elles sont créatives et donnent lieu à de nouvelles situations de conception.

Mots-clés : Science - Design - Figure - Posture - Dramaturgie - Interdisciplinarité

\section{MISE EN RELATION DISCIPLINAIRE ET DRAMATURGIE DE LA SCIENCE}

Cette étude s'inscrit dans le cadre de l'ANR Descitech ${ }^{1}$ qui s'intéresse à l'association du design aux sciences pour comprendre comment cette collaboration permet de formuler de nouvelles approches des savoirs scientifiques dans un souci d'appropriation par les différents acteurs de la société. Plus précisément, notre recherche vise à comprendre comment les scientifiques déploient plusieurs représentations de la science pour entrer dans des dispositifs de conception. À la suite d'observations et de longs entretiens ${ }^{2}$ avec les acteurs, nous décrivons plusieurs situations d'interaction entre design et sciences et repérons les « figures de science » qui apparaissent et sont mobilisées pour répondre aux contraintes de conception. Cette analyse donne lieu au repérage des formes de dramaturgie de la science qui permettent de nouvelles modalités de collaboration. Notre hypothèse est que les scientifiques en milieu de design modulent leurs postures de façon à permettre non seulement de nouvelles formes de collaboration souples et non limitées à leurs connaissances scientifiques mais aussi une redéfinition de l'institution scientifique et de son rôle dans la société.

Cet article s'intéresse donc aux interactions entre les acteurs dans le cadre de projets de conception rassemblant plusieurs disciplines. Si ces situations sont souvent qualifiées et catégorisées comme pluri-inter-trans-disciplinaires, nous ne prendrons pas parti et préférons l'expression plus générale et non-restrictive de «mise en relation de disciplines ». En effet, nombreuses sont les études qui s'intéressent aux enjeux épistémologiques de ces mises en re-

\footnotetext{
${ }^{1}$ ANR Descitech : programme de recherche de 2014-2018 entre CIME Nanotech Univ. Joseph Fourier, LPS Univ. Paris Sud, ENSCI-Les Ateliers, Codesignlab Télécom ParisTech, UPEC/IRG. https://descitech.hypotheses.org/.

2 Grant McCracken, The long interview, Sage, Vol.13, 1988.
} 
lation disciplinaires ${ }^{3}$ mais peu s'attardent sur la compréhension des rôles que chacun des acteurs y jouent. Comme le montre Lenoir, les recherches menées tentent de catégoriser et de hiérarchiser les relations entre les disciplines scientifiques selon «trois voies d'appréhension [...] celle de l'établissement de "liens entre" les disciplines, [...] celle de l'émergence de nouvelles disciplines à partir du constat d'un "vide entre" les disciplines déjà existantes, [...] et celle, enfin qui promeut une nouvelle [re]structuration, voire la naissance d'une nouvelle conception et d'une nouvelle organisation des savoirs scientifiques. $»^{4}$.

Or, ces considérations d'ordre épistémologique nous semblent omettre, notamment dans le cas de projets de conception qui s'appuient sur plusieurs disciplines, qu'elles sont représentées par des individus qui revendiquent plus ou moins ouvertement leur rattachement disciplinaire, ou à qui leurs interlocuteurs associent des savoirs et savoir-faire scientifiques considérés comme essentiels à la conception. Il nous semble ainsi intéressant de croiser la réflexion épistémologique en regardant ce qu'elle devient sur le terrain du design, par le biais des acteurs et de leurs interactions et de ce que ces interactions disent des rapports entre design et sciences. Ici, nous nous intéressons à ce que les scientifiques « retiennent » ou font évoluer de leurs postures « scientifiques » dans un milieu de design. Autrement dit, en suivant les « expressions de science » sur le terrain, nous souhaitons comprendre comment les sciences sont mobilisées dans le discours des acteurs du binôme design/sciences, afin de mieux cerner ce qu'une situation de conception fait évoluer de la représentation et de l'usage des sciences.

En nous appuyant sur l'analyse et la théorie goffmanienne de l'interaction, nous portons notre regard sur les personnes qui, dans l'interaction, entrent dans un processus d'influence réciproque au travers de " rôles » qui se forment et se déforment en fonction des situations d'interaction, notamment lorsqu'il s'agit de rencontres disciplinaires. Nous souhai-

\footnotetext{
${ }^{3}$ Guy Berger, « Opinion et réalités », in Léo Apostel, Guy Berger, Asa Briggs et Guy Michaud (dir.), L'interdisciplinarité. Problèmes d'enseignement et de recherche dans les universités, Paris, Organisation de coopération et de développement économique, Centre pour la recherche et l'innovation dans l'enseignement, p. 21-69, 1972 ; Jérôme Bourdon, « L'interdisciplinarité n'existe pas », Questions de communication, n 19, p. 155-170 ; Georges Gusdorf, «Interdisciplinaire (connaissance) », in Encyclopedia Universalis, Vol 8, p. 1086-1090, 1e éd., 1968 ; Georges Gusdorf, « Passé, présent, avenir de la recherche interdisciplinaire », in . In Léo Apostel, Jean-Marie Benoist, Tom Burton Bottomore, Kenneth Ewart Boulding, Mikel Dufrenne, Mircea Eliade, Ceslo Furtado, Georges Gusdorf, Daya Krishna, Wolfgang J. Mommsen, Edgar Morin, Massimo Piattelli-Palmarini, Mohammed Allal Sinaceur, Stanislav Nikolaevitch Smirnov, Jun Ui (éd), Interdisciplinarité et sciences humaines, Paris, Unesco, Vol. 1, p. $31-51$; Julie Thompson Klein, Interdisciplinarity : History, theory and practice, Wayne State University Press, 1990 ; Joseph J. Kockelmans, Interdisciplinarity and Higher Education, Philadelphia, The Pennsylvania State University Press, 1979 ; Jean-Paul Resweber, La méthode interdisciplinaire, Paris, Presses universitaires de France, 1981 ; Jean-Paul Resweber, «Les enjeux de l'interdisciplinarité », Question de communication, $\mathrm{n}^{\circ} 19, \mathrm{p}$. 171-200, 2011.
}

4 Yves Lenoir, «L'interdisciplinarité : aperçu historique de la genèse d'un concept », Cahier de la recherche en éducation, 2, p. 227-265, 1995. 
tons ainsi appliquer la théorie de l'agir dramaturgique 5 qui montre que dans l'interaction, « l'acteur doit agir de façon à donner, intentionnellement ou non, une expression de lui, et les autres à leur tour doivent en retirer une certaine impression $»^{6}$. Les individus en présence se co-construisent sur la base d'une analyse mutuelle et forgent une définition de la situation où le spectateur [récepteur] répond à l'acteur [locuteur] en adoptant une conduite déterminée et inversement ${ }^{7}$.

Nous souhaitons donc repérer les « rôles » que les scientifiques incarnent en situation de conception au travers de « figures » qui découlent de ces représentations (dramaturgiques) des sciences. En effet, la figure est le résultat d'un travail de figuration passant par une opération de sélection, de caractérisation et de transformation d'éléments d'expérience. Il s'agit d'un travail formel ancré dans l'expérience qui crée un plan autonome par rapport à celle-ci afin de le recomposer. La figure est donc représentative mais également créative et peut donner lieu à de nouvelles situations de conception. Notre étude observe ce travail de figuration à l'œuvre chez les scientifiques, plus particulièrement dans le cadre de formations au design et aux sciences en simultané.

Comme le montre B. Bensaude-Vincent ${ }^{8}$ dans son analyse de l'évolution du rapport science/public au cours des trois siècles précédents, la représentation du scientifique a évolué. D'abord, le savant universel des XVII-XVIIIe siècle, "honnête-homme" incarné par des personnalités telles que Diderot ou encore Voltaire, dont la mission est de répandre les lumières. Ensuite le "surhomme" du XXe siècle dont l'expertise s'accroît très vite dû aux deux guerres mondiales, il a le pouvoir de tuer (pendant les guerres) et de sauver (évolution de la médecine), il fascine et effraie en même temps. Enfin, "l'hyper-spécialiste" du XXIe, en dehors du monde, irresponsable parce que soumis à des financements privés, et donc à des résultats privés, qui peut renvoyer à la figure du "savant-fou" présente dans la littérature du XIXe siècle et dont le savoir se transforme en pouvoir (Noiray, 1998). Cette évolution marque l'élargissement croissant d'un fossé entre le public et les scientifiques. Nous comprenons ainsi que les scientifiques doivent aujourd'hui faire face à de nouveaux enjeux : un impératif de se réancrer dans le monde et la société afin de produire ce que B.Bensaude-Vincent appelle une «science citoyenne ", mais également montrer un modèle responsable et éthique des sciences vis-à-vis des futurs : il ne peut plus être question de la "science pour la science", mais de la science pour le « bien commun ». Dans la lignée des figures historiques dépeintes par B.BensaudeVincent, nous avons souhaité regarder concrètement la façon dont la science est figurée dans les différents terrains de conception de notre étude.

Dans une première partie nous décrivons les terrains d'observations et les méthodes en expliquant notamment comment nous avons pris en filature sur plus de trois ans, deux pro-

\footnotetext{
5 Jürgen Habermas, Jean-Marc Ferry, Jean-Louis Schlegel, Théorie de l'agir communicationnel, Tome 1, Rationalité de l'agir et rationalisation de la société, Tome 2, Pour une critique de la raison fonctionnaliste, 1988 ; Erving Goffman, La mise en scène de la vie quotidienne, Tome 1, La présentation de soi, Paris, Minuit, p. 23, 1973.
}

${ }^{6}$ Ibidem, p. 12.

${ }^{7}$ Ibidem, p. 18.

8 Bernadette Bensaude-Vincent, «Splendeur et décadence de la vulgarisation scientifique », Questions de communications, ${ }^{\circ} 17$, p. 19-32, 2010. 
fesseurs de physique, auprès desquels nous avons recueilli les récits de vie, récits qui ont fait émerger des « figures » de scientifiques. Une deuxième partie répertorie, définit et analyse les figures repérées dans l'étude : le professeur d'université, l'honnête-homme, l'expert, le chercheur et le scientifique-créateur. Nous définissons enfin, en conclusion et dernière partie de cet article, les concepts de figure et de posture en regard des résultats de notre étude.

\section{DES PHYSICIENS PRIS EN FILATURE :}

L'étude longitudinale a été organisée comme une filature de deux professeurs de physique dans divers contextes de conception / design par les auteures de l'article.

Le premier physicien (P1), professeur des universités, propose depuis sept ans à des populations diverses (écoles de design, écoles d'ingénieurs, cursus universitaires de physique) de découvrir des principes de physique fondamentale dans des workshops créatifs en mettant en œuvre ces principes afin de les symboliser, de les explorer esthétiquement ou de les expliquer.

Le deuxième physicien ( $\mathrm{P} 2)$, également professeur des universités, s'intéresse à la notion de mouvement en physique, intérêt qui l'a conduit à développer un dispositif d'expérimentation et d'apprentissage s'appuyant sur les capteurs présents dans un appareil très populaire : le smartphone, afin de mesurer diverses données nécessaires pour comprendre le mouvement. Sa recherche sur le geste et le mouvement, ainsi que l'usage de ce dispositif l'entraînent à la rencontre des designers et des artistes qui expérimentent sous un angle plus sensible ces questions, afin de faire ressentir le mouvement différemment que par des mesures de physique.

Cette double « filature » nous a entraînées sur quatre terrains principaux sélectionnés pour leur variété d'approche de la conception. Il ne s'agissait pas de faire des portraits de scientifiques, mais de mener une enquête qui consiste à saisir un processus collectif activé par la production d'une figure scientifique.

\section{Le physicien 1 : P1}

Le premier terrain (T1) s'est déroulé en 2016 à l'ENSCI-Les Ateliers, une école de design industrielle, sur une durée de 6 mois. L'école fonctionne par ateliers encadrés par des designers professionnels et invite les étudiants à expérimenter et à apprendre en faisant. Dans le cadre de l'atelier « Design et Recherche », co-encadré par un designer et un chercheur invité, le physicien P1 a proposé un atelier autours des questions du microscopique, de l'invisible et de la physique, intitulé " voir l'invisible ». Cette collaboration entre pensée académique et pratique de design a donné lieu à des formats de projets divers : vidéo expérimentale, performance théâtrale, dispositif pédagogique et objet fonctionnel.

Le deuxième terrain (T2) est celui de l'Institut Villebon-Charpak auprès d'étudiants qui préparent une licence généraliste « Sciences et Technologies ». Dans ce contexte, P1 intervient lors d'ateliers de création et d'innovation avec un professeur de gestion (40 heures décembre 2016 et en décembre 2017). Les groupes d'un peu plus de trente élèves incarnent 
des « spin-off $»^{9}$ d'entreprises multinationales réelles soumises à des écosystèmes dynamiques de parties prenantes économiques, sociales et juridiques. Les groupes affectés à une entreprise doivent créer de nouveaux produits sur la base d'une découverte scientifique fictive (la lévitation pour le premier workshop et l'invisibilité pour le second) mais à base scientifique sérieuse.

\section{Le physicien 2 : P2}

Le troisième terrain (T3) a eu lieu de nouveau en 2016 à l'ENSCI-Les Ateliers auprès des étudiants. Cet atelier plus court que le précédent a duré une semaine. Intitulé «Smartphones et mouvement : gestes captés », il visait notamment à désacraliser le smartphone pour ne plus le considérer comme un archétype contemporain de la vie quotidienne, mais comme un moyen de captations diverses, comme un médium ou un outil capable de percevoir de manière sensible le mouvement et/ou les gestes. L'atelier a réuni un designer professionnel, une danseuse, une équipe de l'IRCAM spécialiste du traitement du signal et enfin le physicien (P2) pour encadrer et servir de ressource pour le développement des projets des groupes d'étudiants.

Le quatrième terrain (T4) se déroule au CRI, Centre de Recherche Interdisciplinaire, qui héberge le master AIRE co-créé par les université Paris Descartes et Paris Diderot. Il est dédié à l'apprentissage des sciences et des pratiques numériques dans une démarche d'apprentissage par le faire, d'expérimentation et de conception. P2 y est professeur associé. Le thème général de ce master sur la transformation numérique de l'éducation l'amène, depuis 2014, à donner des ateliers sur les questions de gestes et de mouvements en s'appuyant sur « le smartphone pocketlab » que nous avons décrit plus haut, ateliers qui peuvent ouvrir des questions comme celle du numérique sans écran par exemple.

\section{Méthodes :}

Derrière l'apparente facilité de ces terrains, reposant sur la bonne collaboration de toutes les parties concernées, il a été en fait très difficile de saisir certaines postures qui non seulement ont déjoué les attentes des observateurs mais aussi parfois celles des acteurs euxmêmes. En effet, c'est bien dans l'interaction avec des groupes d'interlocuteurs très divers que les deux professeurs de physique découvrent de nouvelles facettes de leurs contributions possibles. C'est en particulier ce que $\mathrm{P} 2$, « immergé » dans différents milieux, pour reprendre l'expression de Mathias Béjean et Jean-Claude Moisdon ${ }^{10}$, exprime : un parcours initiatique où il se laisse surprendre non seulement par les questions mais aussi par les attentes sur ce

\footnotetext{
${ }^{9}$ Nous employons le terme de « spin-off » pour parler des projets d'entreprises fictives, dérivées d'entreprises multinationales connues. Elles s'appuient sur ce qui fait le succès de ces multinationales pour développer une dimension connexe.

10 Mathias Béjean et Jean-Claude Moisdon, « Recherche-intervention et Living Labs, une même lignée de « recherche immersives »? », in Annales des Mines-Réalités industrielles, $\mathrm{n}^{\circ} 2$, p. $54.59,2017$.
} 
qu'est sa «place ». Nous reprenons ce terme de place de Jeanne Favret-Saada ${ }^{11}$ qui décrit le fait que les chercheurs peuvent se faire remettre à leur place par leurs interlocuteurs :

" Prendre place ou plutôt se voir "assigner" une place dans un système actanciel permet de saisir de l'intérieur le type d'actions, de paroles et d'affects que cette place impose et autorise à ses “occupants". On ne réalise l'existence d'un système de places que si on se cogne à ses limites, si on se fait "remettre en place" ".

Ici, ce qui nous intéresse, ce sont les multiples «places » assignées aux, ou endossées par les physiciens et la façon dont cette place définit en filigrane l'institution scientifique.

En plus de l'observation nous avons récolté et analysé les supports utilisés par les physiciens pour communiquer leur pratique à un tiers, les présentations vidéo-projetées notamment. De nombreux entretiens ont également ponctués cette enquête, qui, à différents moments de la recherche, ont révélé des prises de conscience. En nous appuyant sur l'analyse du discours décrite par Charaudeau et Maingueneau, à savoir que, dans toute situation de communication, le locuteur compose son discours par rapport à l'interlocuteur, qu'il a luimême construit par une interprétation de signes que lui renvoie l'interlocuteur, ainsi que par rapport à la situation même où se déroule l'acte de communication ${ }^{12}$, nous avons analysé les récits des physiciens en suivant la grille de lecture suivante : comment le physicien parle de lui-même - comment le physicien parle de lui par rapport au récepteur - comment il parle du récepteur - comment il parle de lui par rapport au contexte - comment il parle du contexte. Ces entretiens ont permis aux physiciens de mentionner des postures en rapport avec des contextes particuliers. Dans les discours, nous avons ainsi tenté de repérer toutes les « figures » du scientifique immergé en design, figures qui engagent la personne de façon authentique mais aussi flexible et ajustable à l'ensemble des enjeux de création.

\section{LES FIGURES À L'OEUVRE DANS LA DRAMATURGIE SCIENCE ET DESIGN}

L'incarnation de figures et le changement de posture en fonction du public qu'opèrent les scientifiques ne se fait pas sans difficulté et de manière instantanée. Nous observons en effet dans les récits de vie que nous avons recueillis auprès des physiciens qu'il y a un avant et un après leur rencontre avec les sciences de la conception. Par exemple, en parlant d'un projet de recherche sur le mouvement et les capteurs présent dans un smartphone, P2 parle de : " "contamination" par le design », contamination que l'on peut définir comme étant une « altération des caractéristiques propres à une chose ${ }^{13}$. Ainsi, d'après P2, la rencontre avec le design provoquerait une altération de son regard. Cette rencontre influence en particulier la façon dont il va comprendre le smartphone. Son regard de physicien l'avait déjà

\footnotetext{
11 Jeanne Favret-Saada « Glissements de terrains. Entretien avec Jeanne Favret-Saada », $V a-$ carme, 28, p. 4-12, 2004.

12 Patrick Charaudeau, Dominique Maingueneau, Dictionnaire d'analyse du discours, Seuil, 2002.
}

13 http://www.cnrtl.fr/definition/alt\%C3\%A9ration, site consulté en Juillet 2018. 
conduit à le voir comme un appareil de mesure. Mais avec les designers, il va le replacer dans un contexte d'usage, dans son rapport à la main, et il va s'intéresser au geste. La « contamination au design » lui ouvre de nouvelles perspectives sur la manière dont il regarde cet objet. « Donc la question du mouvement, la question du geste, est naissante chez moi. Et cette question du mouvement et du geste est naissante, et pas naturelle pour un physicien, et notamment un enseignant de physique. Donc là, je crois qu'elle est nettement influencée par le design. Elle vient d'ailleurs de là. »»

Ainsi, comme le montre Resweber, dans la mise en relation de discipline, le sujet « en vient à adopter un autre regard qui subvertit le sien propre et peut l'amener à se position-

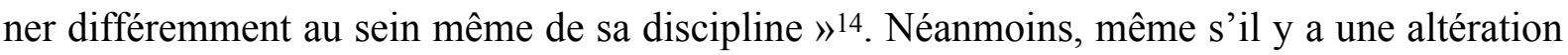
par enrichissement du concept de physique de mouvement par la notion de performativité gestuelle issue du design, d'après le physicien, la « contamination » n'affecte pas les méthodes scientifiques, mais l'entraîne plutôt à intervenir de façon inattendue dans différentes institutions. En effet, l'analyse des entretiens et des présentations faites dans les différents ateliers avec les designers/concepteurs montre, qu'en réalité, l'intervention des scientifiques en milieu de conception adoptent de nouvelles postures, reflétant plusieurs figures de la science auprès des divers publics.

« Il y a trois choses finalement, je peux me présenter :

- comme prof

- comme physicien

- comme porteur d'une méthode scientifique et d'un esprit critique

et je peux être :

- prof en étant ancré sur la méthode scientifique et l'esprit critique

- prof en étant ancré sur la physique

- physicien uniquement comme chercheur à égalité avec tous les participants. »

Nous allons voir dans la suite de l'article à quelles figures correspondent ces postures énumérées par l'un des scientifiques lors des entretiens et ce qu'elles représentent de la science.

\section{a. La figure du professeur des universités : La science comme démonstration d'une vérité ex cathedra}

Pour commencer, nous avons demandé aux physiciens de nous parler des enseignements qu'ils donnent tous deux en université, afin de comprendre leur " point de départ » avant les premières collaborations avec des designers. Nous leur avons demandé de nous décrire le plus précisément possible le déroulement des cours théoriques, et/ou des travaux dirigés (TD), les attentes des élèves, leurs attentes, les supports/médias employés, etc. Tous deux catégorisent ces séances « classiques » de plusieurs façons :

- la position de l'enseignant, « devant », « au tableau debout », par rapport à celle des étudiants, « assis dans une structure classique de classe»;

- le modèle général d'apprentissage « est très classique et top-down »;

14 Jean-Paul Resweber, La méthode interdisciplinaire, Paris, Presses universitaires de France, 1981. 
- les moyens : « rien que du très classique : explications, pédagogie, exemples, résolution de certains exos au tableau, puis ensuite en travaux dirigés, travail sur des exercices pour mettre en application »;

- la posture des étudiants : « souvent assez passive et « classique », peur de l'examen, scolaires, peu d'engagement au-delà du cadre proposé (par exemple à travers une prise d'initiative, un projet personnel, etc.) ».

Cette posture réintervient dans les ateliers avec des designers. Ainsi, P2 remarque que les professeurs peuvent se retrouver « dans un mode "on présente et on écoute" en particulier au tout début des workshops ». Il précise d'ailleurs à propos de l'atelier qu'il a accompagné à l'ENSCI (terrain 3) : «c'est le seul moment dans la matinée du lundi où ça arrive ».

Les objectifs des enseignements reposent ainsi sur « le transfert de connaissances et de méthodes » pour répondre aux attentes des étudiants qui, selon P1, veulent « acquérir des connaissances et savoir-faire disciplinaires sur un des champs de la physique, pour réussir leurs examens ». Il remarque également qu'un polycopié du cours est souvent attendu par les étudiants mais pas toujours donné par les enseignants, qui, de leur côté, attendent « une prise de recul sur la discipline » et une prise d'autonomie de la part des étudiants pour " résoudre ensuite des problèmes plus complexes ». Concernant les TD, P1 raconte que les étudiants doivent suivre un protocole qui se trouve dans un manuel et mener l'expérience « souvent très cadrée ». L'enseignant est « en général dans un mode " circulation », il aide s'il y a des blocages et pousse plus loin ceux qui n'ont pas de difficultés.

La «panoplie du physicien » comme l'exprime P2, et la figure « classique » de l'enseignant de physique qu'ils incarnent, qu'il définit comme ayant « un espèce de plan cohérent de transmission de connaissances », permet ainsi d'atteindre aisément les deux premiers niveaux d'apprentissage que décrit Bateson ${ }^{15}$ : (1) apprendre la bonne réponse, (2) apprendre à se comporter par rapport à la situation, ici la salle de classe / la salle de TD, c'est le « « curriculum caché » de ce que signifie être étudiant : comment plaire à l'enseignant, comment réussir les examens, comment appartenir à un groupe, etc. $\rangle^{16}$. En revanche, et cela est très présent dans les discours de $\mathrm{P} 1$ et $\mathrm{P} 2$, les étudiants ont des difficultés à sortir du carcan scolaire. La relation verticale entre l'autorité de l'enseignant, qui possède le savoir, avec l'étudiant, ignorant, correspond alors à la figure critique du « maitre-explicateur $»^{17}$ que décrit Rancière à partir du récit de Jacotot. Dans le cas de l'université, les scientifiques sont donc les savants qui disposent du savoir et l'expliquent à l'ignorant en faisant la démonstration de la vérité scientifique auprès des étudiants.

\section{b. La figure de l'honnête-homme : La science comme culture générale}

La figure de l'honnête-homme scientifique apparaît dans au moins deux types de situations : l'introduction de la discipline devant des non-spécialistes et l'usage des comparai-

15 Gregory Bateson, « The logical categories of learning and communication », Steps to an Ecology of Mind, p. 269-308, 1972.

16 Yrjö Engeström, « Expansive learning at work: Toward an activity theoretical reconceptualization », Journal of education and work, 14-1, p. 133-156, 2001.

17 Jacques Rancière, Le maître ignorant, Paris, Fayard, 1987. 
sons faisant appel à une large culture générale, artistique en particulier. Nous avons choisi de nommer cette figure ainsi après la tradition dix-septièmiste. Dans des travaux menés sur les CDroms culturels nous avions montré que cette figure est particulièrement vivace pour vanter les mérites de l'apprentissage par une forme de "picorage", de curiosité, organisées autour des personnalités importantes du monde des arts et des sciences et de leurs réalisations ${ }^{18}$. On retiendra essentiellement deux traits qui semblent pertinents pour la compréhension des situations qui nous intéressent. Par exemple sur l'invisibilité, P1 fait l'inventaire des découvertes scientifiques sur l'infiniment petit, présente les grandes figures (prix Nobel) qui sont les artisans de ces découvertes, et montre également des galeries d'images qui nous sont aujourd'hui accessibles. L'objectif de ces présentations est de donner une culture générale sur la discipline fondée sur un rapide historique et aussi d'introduire une culture visuelle puisque les images présentées, mystérieuses et fascinantes, sont considérées par leur présentateur comme autant de sources d'inspiration. La dimension visuelle est d'ailleurs présentée comme introduisant un lien fort avec le design. P2 au cours de son encadrement de workshop, fait lui appel à de nombreuses comparaisons artistiques. Il écrit d'ailleurs des articles sur le rapport sciences et art, comme par exemple au sujet du scientifique Mark Pauly qui travaille sur les « caustiques »: « Des caustiques sont par exemple ces courbes de lumière complexes et dansantes qui se dessinent sur la table derrière un verre d'eau au soleil ${ }^{19}$ et le compare à Pierre Soulages : " dans les deux cas, il s'agit de travailler les détails d'une surface pour installer la lumière dans 1 'espace $»^{20}$. Pour P1, il s'agit de présenter des fresques historiques et ses héros, pour P2, de tisser des liens avec les différentes formes de création humaines. Dans les deux cas, il s'agit aussi d'être sensible aux questions de perception, ce qui ouvre de fait un passage avec le design.

Cette figure de l'honnête homme s'oppose à l'érudit : « il est ouvert à toutes les questions qui peuvent intéresser un esprit distingué, il ne veut point passer pour un spécialiste $»^{21}$. Les interventions des deux professeurs dans les classes de design précisent en effet :

- qu'il ne s'agit pas de transformer les élèves designers en élèves de physique ;

- qu'il s'agit néanmoins de comprendre certains enjeux de sciences : par exemple, lier les différents microscopes aux différents types d'images qu'ils permettent ;

- qu'il s'agit aussi de faire preuve de curiosité.

\footnotetext{
18 Annie Gentès, « Renaissance Man, « honnête homme » and self-made man : lectors in machina in the Electronic Culture », in Nathalie Roelens et Yves Jeanneret (dir.), L'imaginaire de l'Écran / Screen Imaginary, Amsterdam, New York, Editions Rodopi, p. 207-219, 2004.

$19 \mathrm{https}$ ://theconversation.com/quand-les-outrenoirs-de-pierre-soulages-dialoguent-avec-lascience-70608, site consulté en juillet 2018.

\section{Ibidem.}

21 Jean-Claude Chevalier, «Les « Entretiens d'Ariste et d'Eugène » du Père Bouhours, soit la littérature et l'idéologie », in Michèle Duchet et Michèle Jally (éd.), Langue et langage de Leibniz à l'Encyclopédie, 10-18, p. 538-542, 1977.
} 
Analysés par Jean-Claude Chevalier, Les « Entretiens d'Ariste et d'Eugène » du père Bouhours sont considérés comme représentatifs du discours de l'honnête homme au XVIIe siècle. Au-delà de l'intérêt historique et sociologique de l'ouvrage du père Bouhours, on ne peut manquer d'être frappé par les similitudes entre la description de l'accès à la culture de l'honnête homme au XVIIe et le discours tenu par les physiciens :

«Chaque science a un objet particulier où elle s'arrête. [...] Cependant étant limitées comme elles sont, elles ne laissent pas d'être longues à apprendre : la vie est trop courte pour en bien savoir une seule ; et ce qu'il y a de fâcheux, c'est qu'on ne les apprend qu'avec peine. [...] La devise n'a rien de tout cela. [...] Elle est courte parce qu'elle ne prend que la fin des choses : elle choisit ce qu'il y a de plus rare dans la nature, de plus curieux dans les Arts, de plus remarquable dans l'Histoire et de plus exquis dans les Auteurs. La science des devises est courte encore parce qu'elle instruit en un moment. Il ne faut que regarder pour apprendre : une vue simple, mais une vue pénétrante est toute la lecture et toute la méditation qu'elle demande $»^{22}$.

Dans l'idéal aristocratique de l'honnête homme, la connaissance n'est pas assujettie à une nécessité de conquête sociale. Elle est louée pour elle-même. Elle est un outil de la vie mondaine et de son moteur, la conversation ${ }^{23}$. L'honnête homme aux manières raffinées exige un public. Il manifeste ses qualités dans la conversation. Il est ainsi intéressant que P2 ait publié 17 articles dans The conversation. Le partage des connaissances avec les étudiants est placé sous le signe du plaisir, plaisir de la connaissance pour elle-même et par elle-même. L'honnête homme fait aussi preuve de qualités de modestie et de noblesse de cœur. Il peut sembler étrange de souligner ces qualités, mais les physiciens précisent à de nombreuses reprises qu'ils ne sont pas là pour impressionner avec leur savoir, et les observations confirment qu'ils font tout pour mettre à l'aise les étudiants par rapport aux savoirs en physique. De nombreuses expressions ponctuent leur discours : « Ça n'a [pas] plus de prétention que ça non plus... », « on n'est pas là pour... ", « moi je n'interviens pas comme physicien », « moi la seule chose que j'ai faite pour ça c'est faire les courses. Aller acheter de la mousse et euh des choses comme ça ».

\section{c. La figure de l'expert : La science comme réservoir de connaissances utili-} sables

Les discours à propos des deux ateliers donnés à l'ENSCI (T1 et T3), montrent que $\mathrm{P} 1$ et P2 s'y présentent comme « physicien » " à égalité avec tous les participants " (à savoir, les autres intervenants, designers, scientifiques, voire les étudiants (en particulier ceux qui ont déjà plusieurs années de design). Ils se qualifient notamment de « ressource », posture qui renvoie selon nous à la figure de l'expert.

En effet, $\mathrm{P} 1$ et $\mathrm{P} 2$ remarquent, à juste titre que, si les designers ne comprennent pas exactement les lois de physique de tel ou tel phénomène, ils sont capables d'en éprouver les effets et de s'en emparer pour concevoir. Nous avons d'ailleurs également recueilli cela dans

\section{Ibidem.}

${ }^{23}$ François Duc de La Rochefoucauld, « De la conversation », in Réflexions, ou Sentences et maximes morales de La Rochefoucauld, Lefèvre, p. 146-150, 1844. 
les discours des différents designers avec lesquels nous nous sommes entretenus. L'une d'entre eux s'est notamment qualifiée « d'imposteure » à l'égard de ses connaissances qui ne sont pas scientifiques mais reposent sur un apprentissage par l'expérimentation jusqu'à obtenir l'effet désiré. La relation avec les scientifiques qu'elle décrit consiste alors à prédire un résultat, à partir de calculs qu'elle-même n'est pas capable de faire, ou bien à résoudre un problème comme « comment produire de l'énergie avec des e-textiles et le mouvement ». Dès lors, et comme le remarquent $\mathrm{P} 1$ et $\mathrm{P} 2$, le contrat passé entre les physiciens et les designers repose sur la fonction de validation et de vérification que telle ou telle projection ou idée, est « possible» ou « plausible » d'après les lois de physique.

La posture des physiciens semble dépendre ainsi de la figure d'expert, dont l'origine latine, expertitus, fait référence à celui qui teste et prouve. Comme le décrit Christelle Rabier ${ }^{24}$, l'expert est quelqu'un qui possède un savoir et savoir-faire technique ou scientifique spécifique, savoirs lui donnant légitimité et droit à donner son avis sur une question technique et/ou scientifique spécifique et relative à cette connaissance. Dans le cadre des projets de conception, la procédure par laquelle ces figures d'experts s'incarnent repose sur le conseil. Autre figure majeure de notre société qu'est le consultant, lequel peut donner son avis, mais ne peut pas prendre de décision ${ }^{25}$. Les étudiants en design exposent un cas ou une situation aux experts-physiciens, et les « consultent » de manière à pouvoir poursuivre et réaliser leur projet. La visée de ces consultations est alors d'accompagner les étudiants dans la réflexion quant à la conduite à tenir vis-à-vis du problème " physique » qu'ils rencontrent ${ }^{26}$. La science est alors représentée comme un réservoir de connaissances qui permet de résoudre des problèmes. Ce qui ne réjouit pas nécessairement les physiciens considérant que cette demande d'expertise « réduit » la portée de leur savoir. P2 déclare en effet : « je peux me comporter comme physicien au sens où je mobilise des connaissances de physique mais ma façon de les présenter est une réduction de capacité d'action comme scientifique lié à la méthode et à l'esprit critique ». Dans ces ateliers de conception (T1 et T3), les physiciens-experts deviennent donc une ressource pour le concepteur et les sciences « fondamentales » deviennent un matériau au même titre que le bois ou le métal à partir duquel les designers explorent une autre manière de dire la science, ou les lois de sciences $^{27}$.

\section{d. La figure du chercheur mentor : La science comme posture épistémologique}

Dans le cas de l'accompagnement du projet au CRI (T4), P2 parle à plusieurs reprises de « la méthode scientifique ». En prenant l'exemple d'une conversation avec un des étudiants il explique ce à quoi il fait référence : «c'est comment je l'amène à une réflexion

${ }^{24}$ Christelle Rabier, « Fields of expertise: A Comparative History of Expert procedures » in Paris and London, 1600 to present, Cambridge Scholars Publishing, 2007.

${ }^{25}$ Maela Paul, « L'accompagnement : une nébuleuse », Éducation permanente, 153-4, p. 43-56, 2002.

${ }^{26}$ Alexandre Lhotellier, Tenir conseil : délibérer pour agir, Paris, Seli Arslan, 2001.

27 Bernard Guy, « Ethique et épistémologie : convergence entre la démarche épistémologique (chercher le vrai) et la démarche éthique (cherche le bien) : point de vue des sciences de l'ingénieur », Hal-archives-ouvertes, 2012. 
pour essayer d'avoir une approche de la question différente. Je ne vais pas lui dire que c'est faux parce que je suis physicien et que c'est comme ça, je vais lui dire peut-être on peut se poser la question différemment et même l'expérimenter, ou essayer de le conduire à se dire » si cette question pour moi se pose, comment je la questionne ». Et donc je vais venir plus dans une démarche liée à la méthode scientifique et pas aux contenus et à des ressources qui seraient des ressources de physicien en termes de connaissance. [...] j'ai plutôt essayé de le conduire à avoir une démarche rigoureuse et pour le coup scientifique pour questionner ses propres affirmations dans le cadre de son projet. En restant dans une attitude bienveillante qui essaye d'être critique, mais pas critique au sens où moi je suis critique, mais critique au sens où j'essaye de l'amener à une vision critique de son propre argument ». On observe ainsi une autre posture, qui se distingue de celle du professeur d'université, ou encore de celle de l'expert, en cela qu'elle ne s'appuie ni sur une validation, ni sur du « contenu de physique », mais sur la démarche scientifique. Celle-ci repose sur la posture du mentor, qui est tirée du récit de L'Iliade et l'Odyssée de Homère, dans lequel Mentor, ami d'Ulysse, se voit confier l'éducation de Télémaque pendant le long périple de son père. Mentor joue alors plusieurs rôles qui définissent ce qu'est le mentorat aujourd'hui : une relation entre un individu plus vieux et expérimenté - le mentor - et quelqu'un de plus jeune et de moins expérimenté - le protégé —, relation ayant pour but d'aider à développer la carrière du protégé28. Il est ainsi attendu du mentor qu'il soit « capable de composer avec la complexité, qu'il ait des habiletés relationnelles et possède des compétences reliées à une expertise ${ }^{29}$. Ainsi, parce qu'il est chercheur en physique, qu'il possède une expertise et qu'il sait gérer la complexité par ce qu'il appelle « la méthode scientifique », P2 incarne la figure du chercheur qui prend forme au travers du mentorat des étudiants. Pour P1 et P2 il s'agit de montrer ce qu'est la posture de recherche qui s'appuie sur le doute et la critique au travers d'un questionnement constant des affirmations courantes. Les physiciens incarnent alors la figure du " chercheur » en aidant à ouvrir des questions dont les étudiants vont devoir faire l'expérience pour parvenir au projet souhaité, en les aidant à avoir un recul critique à tous les moments de la conception, en les interrogeant : « comment vous faîtes ? », « vous êtes sûrs ? », « combien disent cela ? ». Ils peuvent donc aider les étudiants à agir en « chercheurs».

Dans le cas des ateliers de l'ENSCI (T1 et T3), les physiciens considèrent qu'ils partagent avec les designers cette posture de recherche. P2 déclare d'ailleurs à ce sujet que « leur propre façon d'être designer, comporte aussi déjà cette dimension critique ». Ils distinguent néanmoins les résultats de la recherche scientifique des productions de design en déclarant, par exemple à propos du travail d'un designer : « ça ne permet pas l'analyse scientifique derrière bien sûr, puisque là il vient de la court-circuiter complètement. [...] Plutôt que de se soumettre à la contrainte physique pour considérer ces données, il se soumet à la contrainte de la perception humaine. [...] Il vient considérer ces données avec comme seule idée qu'elles fassent sens pour la perception et pas pour le scientifique ». Le design n'apporte, selon eux, rien à la recherche en physique en tant que telle, car elle précède les projets de conception. $\mathrm{P} 2$ reconnaît : « l'impression que j'ai, c'est que quoi qu'on fasse derrière comme représentation des données, on va toujours en passer d'abord par un traitement physique et on va inscrire ces données dans le formalisme qui est celui de la description du mouvement et ensuite une fois

${ }^{28}$ Kathy E. Kram, «Improving the mentoring process », Training \& Development Journal,
1985 ; Daniel Jacob Levinson, The seasons of a man’s life, Random House Digital Inc, 1978.

29 Renée Houde, Des mentors pour la relève, PUQ, Éd. Revue et augmentée, 2009. 
qu'on aura fait ça alors on pourra faire ce qu'on veut en termes de visualisation, de sonification, de mise en lumière, d'associer un signal à ce mouvement, etc. ». Ainsi, dans une expérience précédente menée entre le CEA de Grenoble et l'ENSCI, les résultats reposent davantage sur des scénarios prospectifs d'applications des découvertes scientifiques ${ }^{30}$. Néanmoins, la démarche de conception et la démarche scientifique ont des traits communs qui permettent aux physiciens de représenter la dynamique de la recherche comme partagée entre design et science.

\section{e. La figure du scientifique-créateur : La science comme espace extensif par processus métaphorique}

Les étudiants pointent du doigt le fait que les designers et les différents acteurs présents sur les divers terrains, autres que P1 et P2, dévoilaient leur discipline « source» dans les exemples qu'ils emploient. Nous retrouvons également cela dans le discours de P2 lorsqu'il nous raconte avoir " rajouté l'idée des vibrations » pendant la phase de brainstorming d'un des groupes de l'atelier «Smartphone et mouvement : gestes captés » (T3), en rebondissant sur l'histoire des matières que les étudiants étaient en train de discuter. Il se rappelle avoir utilisé la métaphore du scorpion pour expliquer la manière dont le smartphone capte les vibrations, métaphore qui a permis d'ouvrir un nouvel espace de réflexion pour le groupe : « Scorpion : ça c'est quelque chose qui leur avait bien plu, et c'est pour ça qu'ils ont gardé le nom d'ailleurs : l'enregistrement des vibrations, lorsque je fais ça ici [tapote sur la table] c'est comme ça que cherchent les scorpions puisqu'ils détectent les vibrations qui arrivent et foncent sur la source de la vibration qui est en général un autre insecte ». Néanmoins, P2 n'amène pas cette référence sans considérer des aspects techniques et physiques de la métaphore qu'il emploie : " par exemple il y avait le problème des scorpions et de la physique associée aux scorpions, ça c'est moi qui leur ai introduit. Et je leur introduis en sachant exactement ce que je fais, et je sais que tout est bon [...] au plan de la physique et que c'est fondé donc je leur propose dans ce cas-là un outil, une connaissance sur laquelle ils vont pouvoir appuyer toute leur démarche $»$.

La métaphore permet ainsi d'ouvrir un champ en le confrontant à un autre. Ce travail de la métaphore permet aussi d'établir un pont entre deux entités : un savoir-source (ici le scorpion) d'une part, et d'autre part l'inconnu - le savoir cible (le projet en devenir des étudiants sur la notion de vibration). De cette façon, certains aspects de chacun des deux termes de la comparaison sont éclairés, d'autres sont minimisés, de nouvelles idées émergent et des niveaux de signification plus profonds sont exploités dans le but de créer de nouvelles perspectives sur le monde ${ }^{31}$. Les métaphores démontrent ainsi des manières dont chacun conceptualise le monde. Nous savons en effet que les métaphores sous-tendent notre façon de penser,

${ }^{30}$ Camille Jutant, Julien Bobroff, « Objets de médiation de la science et objets de design. Le cas du projet « Design Quantique » », Communication \& langages, $\mathrm{n}^{\circ}$ 183, p. 9-24, 2015.

31 Gerald G. Cupchik, "The interanimation of worlds: Creative metaphors in art and design ». The Design Journal, 6-2, p. 14-28, 2003. 
de raisonner et d'imaginer la vie quotidienne ${ }^{32}$. En somme, nous expérimentons beaucoup de concept, en particulier abstraits, tels que le temps et les émotions en termes de métaphore ${ }^{33}$.

Ainsi, l'analogie faite entre le concept de vibration et la façon dont perçoit le scorpion est représentative de la manière dont $\mathrm{P} 2$ voit le monde : sous le prisme de la physique. Ses exemples sont donc liés à la physique, mais en se mettant dans la posture de ce que nous appelons un "scientifique-créateur », et en s'immergeant dans le milieu de conception, à « égale de tous les participants » comme le note une des encadrantes d'ateliers du CRI, il fait preuve de créativité. C'est en regardant le monde sous l'angle d'une culture scientifique que P2 ouvre de nouveaux champs d'interprétation et par conséquent de nouveaux espaces de création.

\section{LA DRAMATURGIE DE LA SCIENCE COMME CONDITION D'UNE CONCEPTION PLURIDISCIPLINAIRE.}

Dans le tableau ci-dessous nous récapitulons les différentes figures, les modalités principales d'intervention et les mises en scène de science qui en découlent. Dans cette analyse nous retrouvons certaines figures évoquées par B.Bensaude-Vincent, mais également de nouvelles figures, qui plutôt que de se succéder, vont varier en fonction des situations.

\begin{tabular}{|l|l|l|}
\hline FIGURE & MODALITÉS D'INTERVENTION & DRAMATURGIE DE LA SCIENCE \\
\hline $\begin{array}{l}\text { Le professeur } \\
\text { d'université }\end{array}$ & Donne un cours sur le mode de l'explication & $\begin{array}{l}\text { La science comme démonstration } \\
\text { d'une vérité } \text { ex cathedra. }\end{array}$ \\
\hline L'honnête homme & $\begin{array}{l}\text { Donne une vision d'ensemble de la phy- } \\
\text { sique, son histoire, ses héros, une esthétique } \\
\text { à travers le récit. }\end{array}$ & La science comme culture générale \\
\hline L'expert & $\begin{array}{l}\text { Répond aux questions, fournit les éléments } \\
\text { pour avancer opérationnellement : éviter les } \\
\text { erreurs, certifier la probabilité, et/ou la fai- } \\
\text { sabilité d'un projet. } \\
\text { Permet d'accélérer les projets. }\end{array}$ & $\begin{array}{l}\text { Science comme un réservoir de } \\
\text { connaissances utilisables }\end{array}$ \\
\hline $\begin{array}{l}\text { Le chercheur- } \\
\text { mentor }\end{array}$ & $\begin{array}{l}\text { Cultive des façons d'être au monde : la cu- } \\
\text { riosité, la rigueur, la critique, le doute. } \\
\text { Teste, expérimente. } \\
\text { Agit selon la « méthode scientifique ». }\end{array}$ & $\begin{array}{l}\text { La science comme posture épistémolo- } \\
\text { gique }\end{array}$ \\
\hline
\end{tabular}

\footnotetext{
32 Raymond W. Gibbs, Raymond W. Gibbs Jr., The poetics of mind: Figurative thought, language, and understanding, Cambridge University Press, 1994 ; George Lakoff, Mark Johnson, Metaphors we live by, University of Chicago Press, 2008 ; Joan Turner, " Turns of phrase and routes to learning: The journey metaphor in educational culture », Intercultural communication studies, 7, p. 23-36, 1998.

33 Zoltan Kövescses, Metaphor: A practical introduction, Oxford University Press, 2010 ; Michael Reddy, « The conduit metaphor », Metaphor and thought, 2, p. 258-324, 1979.
} 


\begin{tabular}{|l|l|l|}
\hline $\begin{array}{l}\text { Le scientifique- } \\
\text { créateur }\end{array}$ & $\begin{array}{l}\text { Reformule au travers de la métaphore pour } \\
\text { ouvrir de nouveaux champs. } \\
\text { Regarde le monde sous le prisme de sa } \\
\text { culture scientifique et y tire ses exemples, } \\
\text { qu'il reformule en créant des métaphores, } \\
\text { des analogies qui nourrissent la création. }\end{array}$ & $\begin{array}{l}\text { La science comme espace malléable } \\
\text { par la transposition d'exemple }\end{array}$ \\
\hline
\end{tabular}

Les observations nous ont permis de voir les différentes activités des scientifiques immergés : explications données aux étudiants, retours sur leurs travaux, postures d'écoute, phases d'expérimentation collectives, etc. Au cours de ces observations, de nombreuses postures sont apparues, lesquelles ont été explicitées dans les entretiens avec les physiciens en faisant retour sur leur expérience. Dans cette étude, notre intérêt a moins été de regarder les thématiques scientifiques qui sont abordées dans le cadre des ateliers que d'observer les dramaturgies de la science dans des situations de design. Pour nous, il était important non seulement de considérer le ballet des acteurs et la façon dont ils gèrent leurs espaces de connaissance et de légitimité, mais aussi de considérer ce que ces différentes figures permettent du point de vue de la conception et des mises en relation des disciplines en action. On voit en effet souvent que les scientifiques emploient le design à des visées de vulgarisation, mais nous observons ici ce que les situations de design font aux scientifiques : les designers ont besoin qu'ils adoptent les figures que nous avons énoncées plus haut pour re-formaliser les connaissances scientifiques et ouvrir de nouveaux espaces de conception. Il est alors intéressant de ne pas regarder uniquement des activités mais, dans une perspective communicationnelle, d'observer ces figures, c'est-à-dire les représentations dans les discours qui renvoient à un espace créatif de mise en scène de soi et par conséquent des autres, mais aussi mise en scène des sciences et de la situation de conception.

Il nous semble important à ce stade de revenir sur le terme de figure et ce qu'il nous permet de comprendre du point de vue communicationnel et de la conception. L'origine étymologique du terme est le latin figura pouvant signifier « configuration, forme, aspect, représentation sculptée, mode d'expression (figure de style), manière etc. $\rrbracket^{34}$. La figure peut ainsi être définie comme étant la « forme extérieure d'un corps, d'un être $»^{35}$ ou encore " l'aspect extérieur d'ensemble, relativement caractérisé $»^{36}$. La notion de forme est indissociable de la figure. Parler de figure consiste ainsi à s'intéresser à la composition, au format qui structure les interactions et qui sont co-construites par les acteurs. Dans une analyse des figures, l'accent est mis moins sur le symbolisme que sur les configurations. Comme le dit Jacques Aumont pour le cinéma, « la figure est cette dynamique de la mise en forme, de l'invention des formes dotées d'un pouvoir de symbolisation et dans lesquelles se traduira de la pensée ${ }^{37}$. La notion de figure est de plus utilisée en référence au corps dans l'image cinématographique

${ }^{34}$ http://www.cnrtl.fr/etymologie/figure, consulté en août 2018.

35 https://fr.wiktionary.org/wiki/figure, consulté en août 2018.

36 http://www.cnrtl.fr/definition/figure, consulté en août 2018.

37 Jacques Aumont, À quoi pensent les films, Seguier Éditions, 1996. 
mais aussi dans les études sur le genre ${ }^{38}$. Elle porte en ce sens plus précisément sur les inventions possibles de la figure humaine, du corps en particulier ${ }^{39}$.

Dans le cadre de notre étude, nous nous sommes ainsi intéressées à la figure $\mathrm{du}$ scientifique en reprenant une méthode que nous avions testée sur la notion d'utilisateur final (pour comprendre notamment la diversité des figures d'utilisateurs dans les projets de conception). Dans cette précédente étude, nous avions montré que les figures d'utilisateurs finaux sont des représentations dynamiques qui permettent aux concepteurs de jouer sur des propriétés de l'usage, de reconfigurer l'humain et ainsi d'inventer de nouveaux dispositifs ${ }^{40}$. Ainsi, il ne s'agit pas ici de se contenter de portraits de scientifiques mais de montrer comment, d'une part, ils sont une manière de représenter la science dans son rapport au design et, d'autre part, comment le fait même de la dramaturgie est une mise à distance qui permet la conception. Notre méthode a donc tenté d'isoler des figures construites par les comportements et les discours des scientifiques « immergés » dans des espaces de design, et la façon dont ils configurent les rapports entre sciences et design.

Notre définition de la figure s'appuie ainsi sur la mise en scène de soi étudiée par Goffman mais elle s'en distingue parce qu'elle introduit un plan construit qui se détache des interactions en situation pour se concentrer sur les productions de discours. Notons d'abord, que chacune des figures présentées est reliée à une posture qui est la garante en quelque sorte de l'authenticité (ou de la représentativité) des propos. Comme le rappelle Alain Viala, le terme « posture », en son sens premier, « renvoie à la réalité du corps : [elle] est une attitude, une façon de se tenir, de placer son corps, ses membres $»^{41}$. Affaire de gestuelle et d'attitude du corps, elle est aussi affaire de présentation de soi qui peut passer par le langage. Viala rappelle également que la posture est située, en cela qu'elle est fonction du contexte dans lequel elle prend place, ainsi que du regard d'un destinataire. Comme dans la théorie goffmanienne de l'interaction, il y a un rapport de co-construction dans la prise de « position » (de posture) d'un individu par rapport à un autre dans une situation donnée. Les figures apparaissant dans les discours reposent de façon plus ou moins explicite sur ces postures.

Mais la constitution de la figure est aussi un espace co-construit où des éléments sont choisis, mis en lumière, transformés pour s'ajuster aux situations de façon créative. Nous avons introduit le concept de figure dans l'étude des situations de communication, en tant qu'attention à la construction de soi non pas seulement comme obligation sociale mais comme espace créatif. La dramaturgie de soi permet une grande souplesse dans les interactions en particulier dans la science immersive où, comme l'écrivent Béjean et Moisdon :

38 Patricia Caillé, «Interroger la figure croisée des cinémas et des genres », Nouvelles Questions Féministes, 23-1, p. 44-59, 2004.

${ }^{39}$ Nicole Brenez, De la figure en général et du corps en particulier. L'invention figurative au cinéma, Bruxelles, DeBoeck, 1998.

40 Annie Gentès, The in-discipline of design. Bringing the gap between Humanities and Ingineering, Springer, Design Research Foundation, 2017.

${ }^{41}$ Alain Viala, « Posture », in Anthony Glinoer et Saint-Armand Denis (dir.), Le lexique socius, URL : http://ressources-socius.info/index.php/lexique/21-lexique/69-posture, consulté le 08 juin 2017. 
" la démarche de terrain est ainsi bien davantage une "immersion" qu'une expérimentation. Elle repose, en effet, non pas sur une logique de vérification, de validation ou de falsification d'une théorie portant sur un objet de recherche donné au départ, mais bien sur une logique d'extension de l'objet de recherche, et ce, à partir d'un cadrage théorique moins totalisant $»^{42}$.

En effet, l'ensemble des situations observées a pour finalité la conception de nouveaux espaces de savoir et de nouveaux objets/systèmes. Le fait de jouer sur les différentes figures du scientifique permet de sortir d'un système rigide de références tout en gardant l'horizon d'augmentation des connaissances par la création de nouveaux artefacts ${ }^{43}$. Le concept de figure est donc un moyen de rendre compte d'un écart entre le scientifique, tel qui peut être défini dans un contexte de science, et le scientifique " immergé » qui représente tel ou tel aspect de la science. En somme, les deux physiciens que nous avons suivis, sont à la fois, figure de science, et en même temps, ils figurent la science, dans le sens où ils donnent forme à la science en fonction des contextes d'immersion.

\section{BIBLIOGRAPHIE :}

Bateson Gregory, « The logical categories of learning and communication », Steps to an Ecology of Mind, Chicago, University of Chicago Press, 1972, p. 269-308.

Béjean Mathias et Moisdon Jean-Claude, « Recherche-intervention et Living Labs, une même lignée de " recherche immersives »? », in Annales des Mines-Réalités industrielles, $\mathrm{n}^{\circ} 2,2017$, p. 54-59.

Bensaude-Vincent Bernadette, "Splendeur et décadence de la vulgarisation scientifique », Questions de communications, $\mathrm{n}^{\circ} 17,2010$, p. 19-32.

Berger Guy, " Opinions et réalités », in Léo Apostel, Guy Berger, Asa Briggs et Guy Michaud (dir.), L'interdisciplinarité. Problèmes d'enseignement et de recherche dans les universités, Organisation de coopération et de développement économique, Centre pour la recherche et l'innovation dans l'enseignement, Paris, 1972, p. 21-69.

Bourdon Jérôme, «L'interdisciplinarité n'existe pas », Questions de communication, $\mathrm{n}^{\circ} 19,2011$, p. 155-170.

Brenez Nicole, De la figure en général et du corps en particulier. L'invention figurative au cinéma, Bruxelles, DeBoeck, 1998.

\footnotetext{
42 Mathias Béjean et Jean-Claude Moisdon, « Recherche-intervention et Living Labs, une même lignée de « recherche immersives »? », in Annales des Mines-Réalités industrielles, $\mathrm{n}^{\circ} 2$, p. 54.59, 2017.

43 Bernard Guy, « Ethique et épistémologie : convergence entre la démarche épistémologique (chercher le vrai) et la démarche éthique (cherche le bien) : point de vue des sciences de l'ingénieur », Hal-archives-ouvertes, 2012.
} 
Caillé Patricia, "Interroger la figure croisée des cinémas et des genres », Nouvelles Questions Féministes, n 23-1, 2004, p. 44-59.

Charaudeau Patrick et Maingueneau Dominique, Dictionnaire d'analyse du discours, Paris, Seuil, 2002.

Chevalier Jean-Claude, « Les « Entretiens d'Ariste et d'Eugène » du Père Bouhours, soit la littérature et l'idéologie », in Michèle Duchet et Michèle Jally (éd.), Langue et langage de Leibniz à l’Encyclopédie, coll. « 10-18 », Paris, UGE, 1977, p. 538-542.

Cupchik Gerald G., " The interanimation of worlds : Creative metaphors in art and design », The Design Journal, $\mathrm{n}^{\circ}$ 6-2, 2003, p. 14-28.

Duc de La Rochefoucauld François, « De la conversation », in Réflexions, ou Sentences et maximes morales de La Rochefoucauld, Paris, Lefèvre, 1844, p. 146-150.

Engeström Yrjö, « Expansive learning at work: Toward an activity theoretical reconceptualization », Journal of education and work, $\mathrm{n}^{\circ}$ 14-1, 2001, p. 133-156.

Favret-Saada Jeanne, "Glissements de terrains. Entretien avec Jeanne FavretSaada », Vacarme, n 28, 2004, p. 4-12.

Gentès Annie, « Renaissance Man, « honnête homme » and self-made man : lectors in machina in the Electronic Culture », in Nathalie Roelens et Yves Jeanneret (dir.), L'imaginaire de l'Écran / Screen Imaginary, Amsterdam, New York, Editions Rodopi, 2004, p. 207-219.

Gentès Annie, Renon Anne-Lyse et Bobroff Julien, « Design and Interdisciplinarity : the improbable introduction of " fundamental physics » in a design school », Actes de la Design Research Society Conference de 2016 : DRS 2016, 2016.

Gentès Annie, The in-discipline of design. Bringing the gap between Humanities and Ingineering, Dordrecht : Springer, Design Research Foundation, 2017.

Gibbs Raymond W., Gibbs Raymond W. Jr., The poetics of mind: Figurative thought, language, and understanding, Cambridge, Cambridge University Press, 1994.

Goffman Erving, La mise en scène de la vie quotidienne, Tome 1 : La présentation de soi, Paris, Minuit, 1973.

Gusdorf Georges, «Interdisciplinaire (connaissance) », Paris, in Encyclopedia Universalis, Vol 8, p. 1086-1090, 1er éd. 1968.

Gusdorf Georges, "Passé, présent, avenir de la recherche interdisciplinaire », in Léo Apostel Léo, Benoist Jean-Marie, Bottomore Tom Burton, Boulding Kenneth Ewart, Dufrenne Mikel, Eliade Mircea, Furtado Ceslo, Gusdorf Georges, Krishna Daya, Mommsen 
Wolfgang J., Morin Edgar, Piattelli-Palmarini Massimo, Sinaceur Mohammed Allal, Smirnov Stanislav Nikolaevitch, Ui Jun (éd.), Interdisciplinarité et sciences humaines, Paris, Unesco, Vol. 1, 1983, p. 31-51.

Guy Bernard, « Ethique et épistémologie : convergence entre la démarche épistémologique (chercher le vrai) et la démarche éthique (cherche le bien) : point de vue des sciences de l'ingénieur », Hal-archives-ouvertes, 2012 : https://hal.archives-ouvertes.fr/hal-00736247.

Habermas Jürgen, Ferry Jean-Marc, Schlegel Jean-Louis, Théorie de l'agir communicationnel, Tome 1: Rationalité de l'agir et rationalisation de la société, Tome 2 : Pour une critique de la raison fonctionnaliste, Paris, Fayard, 1987-1988.

Houde Renée, Des mentors pour la relève, Québec, PUQ, Éd. Revue et augmentée, 2009.

Jutant Camille, Bobroff Julien, « Objets de médiation de la science et objets de design. Le cas du projet « Design Quantique » », Communication \& langages, $\mathrm{n}^{\circ}$ 183, 2015, p. 924.

Kockelmans Joseph J., Interdisciplinarity and Higher Education, Philadelphia, The Pennsylvania State University Press, 1979.

Kövescses Zoltan, Metaphor: A practical introduction, Oxford, Oxford University Press, 2010.

Kram Kathy E., «Improving the mentoring process », Training \& Development Journal, v.39, n 4 , 1985, p. 40, 42-43.

Lakoff George, Johnson Mark, Metaphors we live by, Chicago, University of Chicago Press, 2008.

Lejeune Gilles, Gentès Annie et Bobroff Julien «La « conception ancrée » comme mode d'articulation de l'imaginaire et de l'analyse : des "personas" aux "organas" ", Congrès de la SFIC, Actes Vol. 2 : Modèle et stratégies d'acteurs, 2018, p. 70-85.

Lenoir Yves, «L'interdisciplinarité : aperçu historique de la genèse d'un concept ", Cahier de la recherche en éducation, ${ }^{\circ}$ 2, 1995, p. 227-265.

Levinson Daniel Jacob, The seasons of a man's life, New-York, First Ballantine Books Edition, 1978.

Lhotellier Alexandre, Tenir conseil : délibérer pour agir, Paris, Seli Arslan, 2001.

McCracken Grant, The long interview, Sage University Paper, Qualitative Research Methods Series, Vol. 13, 1988. 
Paul Maela, « L'accompagnement : une nébuleuse », Éducation permanente, $\mathrm{n}{ }^{\circ} 153$ 4, 2002, p. 43-56.

Rabier Christelle, «Fields of expertise : A Comparative History of Expert procedures » in Paris and London, 1600 to present, Cambridge, Cambridge Scholars Publishing, 2007.

Rancière Jacques, Le maître ignorant, Paris, Fayard, 1987.

Reddy Michael, « The conduit metaphor », Metaphor and thought, n², 1979, p. 258-324.

Renon Anne-Lyse, Gentès Annie et Bobroff Julien, « How to frame the un-known ? The odd alliance of design and « fundamental physics » in a design school. ", Design and Technology Education : an International Journal, $\mathrm{n}^{\circ} 22.3,2017$, p. 105-115.

Renon Anne-Lyse, de Montbron Adrien, Gentès Annie et Bobroff Julien, "From ANT to Material agency : a design and science research workshop », IASDR 2017 Re-search, Cincinnati, United States, oct. 2017.

Resweber Jean-Paul, La méthode interdisciplinaire, Paris, Presses universitaires de France, 1981.

Resweber Jean-Paul, « Les enjeux de l'interdisciplinarité », Question de communication, $\mathrm{n}^{\circ}$ 19, 2011p. 171-200.

Thompson Klein Julie, Interdisciplinarity : History, theory and practice, Wayne State University Press, 1990.

Turner Joan, « Turns of phrase and routes to learning: The journey metaphor in educational culture », Intercultural communication studies, ${ }^{\circ}$ 7, 1998, p. 23-36.

Viala Alain, " Posture », in Anthony Glinoer et Saint-Armand Denis (dir.), Le lexique socius, URL : http://ressources-socius.info/index.php/lexique/21-lexique/69-posture.

\section{AUTEURES :}

Annie Gentès est Maître de conférence HDR en Science de l'Information et de la communication à Télécom ParisTech. Elle dirige le CoDesign Lab, au sein duquel elle s'intéresse aux nouvelles pratiques de conception qui vont au-delà de la place traditionnelle de l'activité de design. À partir de son travail avec des artistes, des designers, des chercheurs en ingénierie, des scientifiques physiciens et biologistes, elle fonde le concept d'《 indiscipline » pour définir un modèle solide de la conception liant les disciplines (The indiscipline of Design, Springer, 2017).

Justine Peneau est doctorante au Codesign Lab sous la direction de Annie Gentès dans le cadre de la Chaire EXALT en partenariat avec Strate Ecole de design. Elle s'intéresse à la conception à la frontière de plusieurs disciplines et s'attache à comprendre le processus 
d' " inter-objectivation » des sensibilités au travers de l'analyse des divers médias et dispositifs par lesquelles celles-ci s'incarnent et se transmettent en nourrissant, déplaçant, transformant les cultures des diverses parties prenantes. 